\title{
TO PERFORM AND JOIN CLUSTER HIGH AVAILABILITY USING LOAD BALANCING APPROACH
}

\author{
Dr. Nirmla Sharma \\ Asst. Professor, Computer Science, Al-Majaradha, \\ King Khalid University, Abha, Saudi Arabia,
}

\begin{abstract}
A system cluster is a set of connected systems, working organized thoroughly so that in numerous respects they method a distinct workstation. The modules of a cluster are usually, but not continuously, associated to respectively further done fast local area networks. Clusters are typically organized to increase presentation and/or accessibility over that delivered by a distinct computer, however normally being much extra commercial than distinct computers of similar speed or accessibility. The cluster is operating a set of processing nodes so as to whole the allocated work in a least quantity of period by working supportively.
\end{abstract}

The core and significant approach to accomplish such goal is by moving the additional loads from full nodes to idle nodes. A cluster advances performance and/or accessibility over that delivered by a different processor. They are great profitable than other processors of equivalent speed or accessibility. The core notion of cluster computing is that, the cluster performs as a particular system to the consumers. Computer clusters have applicability from minor commercial clusters with rare nodes to some of the fastest supercomputers in the domain. It has tried to describe the notion of Cluster computing (Short-lived History of Architecture, types of Clusters, Features, Instances of Cluster Computing and customs of clusters).

Keywords - Architecture, Clutter, performance and remote

\section{INTRODUCTION (ARCHITECTURE OF A CLUSTER)}

Now its greatest simple method, a cluster is a system including two or additional systems or structures (called nodes) which effort composed to perform requests or implement additional jobs, so that consumers whose practice them, need the hollow that individual a particular structure reacts to them, therefore making an impression of a distinct reserve (simulated device). It' model is named transparency of the structure. As crucial properties for building of these stands is involved advancement, dependability, load balancing and performance.

\section{A. Kinds of Clusters}

High Availability (HA) and failover clusters, these prototypes are constructed to offer an obtainability of facilities and properties in a continuous way over the practice of understood dismissal to the organization. The universal information is that if cluster node fails (failover), requests or facilities may be existed in additional node. These kinds are recycled to cluster database of acute assignments, post, files and request servers [1].

Load balancing, this model allocates received circulation or needs for properties after nodes that route the similar sequencers among technologies that create active the cluster. Entire nodes remain accountable to way instructions. Uncertainty node flops, the needs are reallocated between nodes obtainable. The kind of explanation stands typically recycled on plantations of Web servers (web farm).

HA \& Load Balancing Permutation, by way of its term states, it associations the properties of equally sorts of cluster, thus cumulative, obtainability and scalability of facilities and properties. This cluster outline remains extensively recycled now network, message, broadcast, or ftp servers.

Distributed and Parallel Processing, the cluster model recovers the obtainability and presentation for requests, mostly huge computational works. An enormous computational trade may be separated into minor jobs it is dispersed about locations (nodes), similar an extremely similar supercomputer. It has shared toward subordinate; Beowulf cluster is different kind of at NASA scheme. These clusters remain recycled for systematic calculating or economic examination, usual for lacking of jobs great processing control.

\section{i. High-Availability (HA) or Clusters Failover}

The workstations must a robust leaning to break once least presume, particular period once requirement it greatest. It is uncommon near discover an administrator who not ever expected a mobile request now internal of dawn by miserable update that computer stayed depressed, and it must to drive and difficult solution [2]. 
High Availability has connected straight toward our increasing requirement on systems, as today it has a dangerous part mainly now businesses whose key functionality stands precisely proposal of about calculating facility, like ecommercial, broadcast, network sites, and databases, between others.

A High Availability Cluster has goals near retain obtainability of facilities delivered by System reproducing servers then facilities over terminated hardware and software rearrange. Numerous computer arrangements composed by way of unique, respectively individual observing others and attractive their facilities uncertainty some of them drive flop. Difficulties of organization have requirement of software problem and display additional technologies scheduled system, distinguish whatever facilities are consecutively to do now circumstance and disappointment. Damage the presentation before treating power stand typically suitable, important aim has not break. Here are approximately exclusions, like actual and assignment acute systems [2].

Fault tolerance is attained finished hardware as attack organizations, articles, terminated sheets, entirely linked web systems toward deliver other tracks for breaking connection.

\section{ii. Load Balancing Cluster}

Load balancing between servers and clients portion of complete result volatile and cumulative practice of web and Internet. It is using to improved system ability, refining presentation. Reliable load balancing has presented now a part of complete web introducing and e-commerce scheme. However it may not grow wedged through thoughts individual sources, it should revenue structures and transport into the initiative this method of consuming knowledge attention inside commercial clients [3].

Cluster systems has founded on load balancing incorporate nodes all requirements of customers have dispersed consistently through nodes. The organizations do not effort collected in distinct procedure then sending needs individually it attain founded schedule arranged and procedure [4, 11].

This kind of cluster is particularly recycled through ebusiness and Internet service providers who essential near resolution changes load from numerous contribution requirements in actual period.

Furthermore, cluster to be accessible, essential confirm individually server is entirely operated. Once it does load balancing among servers that have identical facility to react client, it is happening difficulties as unique or extra servers can react and prepare the message are reduced. Thus it has placed component that will create matching among servers and clients organize. However it can place numerous servers arranged unique, and clients perform unique statement.
Common instance can be the Linux Virtual Server, or fair establish DNS load balancer. Component of balance will require a statement, anywhere consumers attempt to create interaction, named Virtual Server (VS), which sends circulation to server in server group. Component must be devoted to performance of software depends upon organization, or maybe system device that association's physical presentation of software create route of the correspondences and load balancing in particular device [9].

It has emphasized about strategic arguments for execution the situation and achievement through load balancing scheduled controlling devoted servers:

The algorithm recycled for load balancing, compelling concern in what way balancing among servers have prepared and once consumer creates application to Virtual Address ( VS ), entire procedure for selecting server and server reaction essential happen clear and invisible to consumer approach equally uncertainty not at all balancing [5].

Make technique to verify condition servers have active then occupied, dynamic message has not sent to server just had abort (retain active). Technique has recycled and created certain consumer retrieving similar server once need it [6].

Load balancing has additional modest send consumer circulation toward other servers. For correct execution, tools it will essential for balancing features equally stable message correct, confirmation of server's redundancy. All of these objects have required supporting scalability of capacity of circulation systems without ultimately develop a block or distinct fact lapse [7, 12].

Algorithms for balancing are unique significant features popular this environment and it will describe two elementary approaches:

\section{B. Least Connections}

Method sends applications to final founded on number of requirements / server associates. For instance, uncertainty server 1 is presently control 50 requests / associates, and server 2 switches 25 applications / associates, next application / association will be routinely focused to second server, since server presently needs / networks dynamic.

\section{Round Robin}

This technique practices the method of continuously straight needs to the next offered server in round approach. For instance, received associates are focused on server 1 , server 2 and then lastly server 3 then server 1 proceeds [8]. 


\section{Problem Statement (Weighted Fair)}

Performance leads essentials load to create on applications of individually and reaction of similar (presentation) For instance, uncertainty server's server 1 has 4 times faster now repairing applications from server 2 to supervisor spaces better problem strength for server 1 and server 2 .

\section{EXPERIMENT APPARATUS (JOINED CLUSTER HIGH AVAILABILITY AND LOAD BALANCING)}

These joint key targets to offer high performance key joined with probability no ensuring acute breaks. Joined cluster has faultless key to Internet Service Providers system requests somewhere connection processes has actual serious and figure 1 shows below how to join both algorithms [5-6].

Important features:

- Resending the applications of node disappointments uncertainties for it.

- Developed value facility stages for usual link requests.

- Clear combination for separate requests and nonclustered composed now distinct virtual network

- Offer an extremely accessible design structure

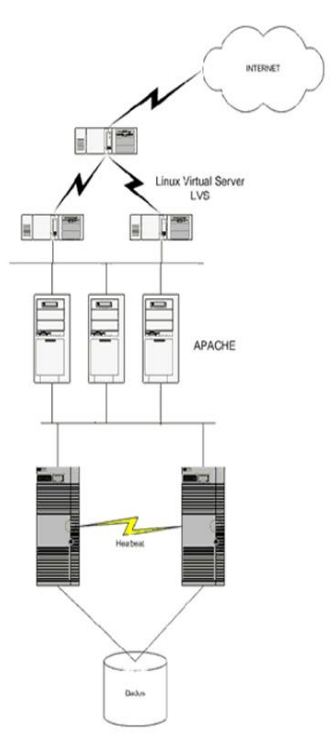

Fig.1. Joined Cluster High Availability and Load Balancing [5-6]

\section{Result AND Discussion (Evaluate the BeOWUlF CLUSTER)}

Individual significant technological progresses of currently has been development of computational performance of PCs (Personal Computers). Certainty that PC market has larger than workstations, permitting reduction value of $\mathrm{PC}$, but its presentation growths considerably corresponding in numerous circumstances, presentation of devoted terminals.

Beowulf cluster has intended through its designers now instruction to happen increasing and high processing control in numerous technical regions instruction near figure controlling and reasonable cloud computing organizations. Of course continuous development of system presentation, and has cooperated method among PCs and workstations, reducing expenses of web processors and individual technologies exposed permitted operating system like GNU / Linux greatly study to effect development of new values of high performance treating now clusters.

An important feature of Beowulf cluster, software has recycled, which has high performance and admiring on important tools, equally an instance it can reference GNU / Linux and Open BSD operating systems which have connected arranged numerous tools to permit processing similar, for example circumstance of PVM and MPI API's. It allows for creating alterations Linux operating system to offer it by original constructions that enabled operation similar requests.

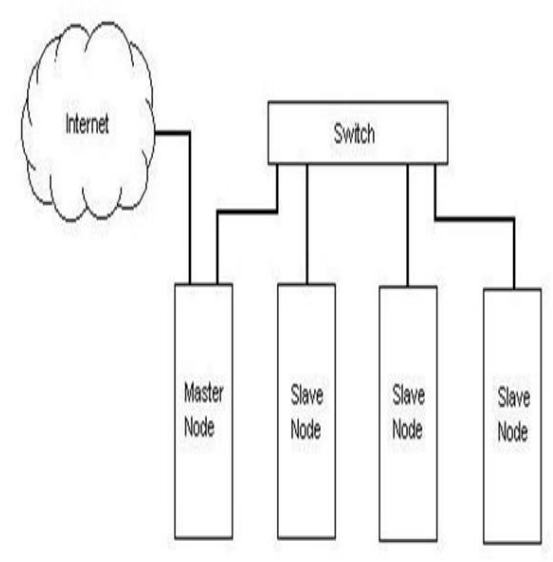

Fig.2. Beowulf cluster retrieved via remote

The organization has separated into supervisor node named front-end (mostly it calls master node), figure 2 shows whose purpose has switch cluster, observing and allocating jobs, performances equally file server and plays connection among operators and cluster. Huge cluster systems may send numerous file servers; system node succeeds not to overcome scheme. Additional nodes have denoted such as clients or back 
ends (fine it calls slave nodes), and have uniquely devoted for treating jobs directed via supervisor node, and there has not requirement keyboards and monitors, then feasibly uniform without procedure of hard drives (remote) boot, besides may be retrieved via remote login (telnet or ssh).

\section{CONCLUSION AND FUTURE WORK}

Arrangements of clusters remain recycled after satisfied is serious or once facilities must to be obtainable then treated for example rapidly and probable. Internet Service Providers (ISPs) or e-business locations frequently need great obtainability and load balancing now an accessible method. The equivalent clusters remain greatly complicated in the Bollywood industry for execution great excellence illustrations and a simulation, reminding that Titanic was reduced inside this stand now Digital Field workshops. Beowulf clusters are recycled now knowledge, engineering and economics to effort on assignments of protein portable, liquid undercurrents, neural networks, genetic analysis, measurements, finances, and astrophysics between others. Researchers, governments and corporations are consuming clusters since they vital to growth their scalability, source organization, obtainability or treating to supercomputing at a reasonable value glassy.

Furthermore, the system is separated into a controller node cluster. For correct result, tools it will essential to must be load balancing features for example stable message form, confirmation of servers and termination.

\section{REFERENCE}

[1] Buyya R. (ed.), (2007)., High Performance Cluster Computing: Systems and Architectures, Prentice Hall.

[2] Demirel Dilek, (2010), High Performance Computing Cluster Architectures, CMPE 511.

[3] Jethwani Kanika, Gaur Surbhi, (2016), A Study on Cluster Computing International Journal of Advanced Research in Computer Science and Software Engineering 6(volume), Issue 9, September , pp. 229-233.

[4] Colajanni M. and E. Casalicchio, (2000) Scalable web clusters with static and dynamic contents., in In Cluster Computing, 2000. Proceedings. IEEE International Conference on.

[5] Zheng Y. H, Guo W. Gao, B. Xu, (2005), Research on Load Balance of Multi Cluster Architecture Based on Business Components Partition, IEEE Proc. of 3rd Int. Conference on Information Technology an Applications.
[6] Buyya Rajkumar Kumar, Cortes Hai Jin, Toni, (2002). Cluster Computing, Future Generation Computer Systems 18 (volume), pp - 5-7.

[7] http://icl.cs.utk.edu/iter-ref

[8] Baker M., Apon A., Buyya R., Jin H., (2006). Cluster Computing and Applications, Encyclopaedia of Computer Science and Technology, August, 45(volume)

[9] Pacemaker - A scalable High Availability cluster resource manager.

[10] http://www.clusterlabs.org/wiki/Main Page

[11] Nayandeep Sran, Navdeep kaur (2013), Comparative Analysis of Existing Load balancing techniques in cloud computing, International Journal of Engineering Science Invention, Vol-2 Issue-1.

[12] Bryhni H., Klovning E and Kure O., (2000), A comparison of load balancing techniques for scalable web servers., Network, IEEE. 